AperTO - Archivio Istituzionale Open Access dell'Università di Torino

\title{
Transactions on Petri Nets and Other Models of Concurrency V
}

\section{This is the author's manuscript}

Original Citation:

Availability:

This version is available http://hdl.handle.net/2318/108499

since

Publisher:

springer

Published version:

DOI:10.1007/978-3-642-29072-5

Terms of use:

Open Access

Anyone can freely access the full text of works made available as "Open Access". Works made available under a Creative Commons license can be used according to the terms and conditions of said license. Use of all other works requires consent of the right holder (author or publisher) if not exempted from copyright protection by the applicable law. 


\section{Lecture Notes in Computer Science}

Commenced Publication in 1973

Founding and Former Series Editors:

Gerhard Goos, Juris Hartmanis, and Jan van Leeuwen

\section{Editorial Board}

David Hutchison

Lancaster University, $U K$

Takeo Kanade

Carnegie Mellon University, Pittsburgh, PA, USA

Josef Kittler

University of Surrey, Guildford, UK

Jon M. Kleinberg

Cornell University, Ithaca, NY, USA

Friedemann Mattern

ETH Zurich, Switzerland

John C. Mitchell

Stanford University, CA, USA

Moni Naor

Weizmann Institute of Science, Rehovot, Israel

Oscar Nierstrasz

University of Bern, Switzerland

C. Pandu Rangan

Indian Institute of Technology, Madras, India

Bernhard Steffen

$T U$ Dortmund University, Germany

Madhu Sudan

Microsoft Research, Cambridge, MA, USA

Demetri Terzopoulos

University of California, Los Angeles, CA, USA

Doug Tygar

University of California, Berkeley, CA, USA

Moshe Y. Vardi

Rice University, Houston, TX, USA

Gerhard Weikum

Max Planck Institute for Informatics, Saarbruecken, Germany 
Kurt Jensen Susanna Donatelli

Jetty Kleijn (Eds.)

Transactions on

Petri Nets

and Other Models

of Concurrency V

胞 Springer 


\title{
Editor-in-Chief
}

\author{
Kurt Jensen \\ Aarhus University \\ Faculty of Science \\ Department of Computer Science \\ IT-parken, Aabogade 34, 8200 Aarhus N, Denmark \\ E-mail: kjensen@cs.au.dk
}

\section{Guest Editors}

\author{
Susanna Donatelli \\ University of Turin \\ Computer Science Department \\ Corso Svizzera 185, 10149 Turin, Italy \\ E-mail: susi@di.unito.it
}

Jetty Kleijn

LIACS, Leiden University

P.O. Box 9512, 2300 RA, Leiden, The Netherlands

E-mail: kleijn@liacs.nl

\author{
ISSN 0302-9743 (LNCS) \\ e-ISSN 1611-3349 (LNCS) \\ ISSN 1867-7193 (ToPNoC) \\ e-ISSN 1867-7746 (ToPNoC) \\ ISBN 978-3-642-29071-8 \\ DOI 10.1007/978-3-642-29072-5 \\ e-ISBN 978-3-642-29072-5 \\ Springer Heidelberg Dordrecht London New York
}

CR Subject Classification (1998): D.2.2-4, D.2, I.6, F.3-4, H.2.3, C.2, C.4

\begin{abstract}
(C) Springer-Verlag Berlin Heidelberg 2012
This work is subject to copyright. All rights are reserved, whether the whole or part of the material is concerned, specifically the rights of translation, reprinting, re-use of illustrations, recitation, broadcasting, reproduction on microfilms or in any other way, and storage in data banks. Duplication of this publication or parts thereof is permitted only under the provisions of the German Copyright Law of September 9, 1965, in ist current version, and permission for use must always be obtained from Springer. Violations are liable to prosecution under the German Copyright Law.

The use of general descriptive names, registered names, trademarks, etc. in this publication does not imply, even in the absence of a specific statement, that such names are exempt from the relevant protective laws and regulations and therefore free for general use.
\end{abstract}

Typesetting: Camera-ready by author, data conversion by Scientific Publishing Services, Chennai, India

Printed on acid-free paper

Springer is part of Springer Science+Business Media (www.springer.com) 


\section{Preface by Editor-in-Chief}

The fifth issue of LNCS Transactions on Petri Nets and Other Models of Concurrency (ToPNoC) contains revised and extended versions of a selection of the best papers from the workshops and tutorials held at the 31st International Conference on Application and Theory of Petri Nets and Other Models of Concurrency, in Braga, Portugal, June 21-25, 2010. It also contains a paper that was submitted to ToPNoC directly through the regular submission track.

I would like to thank the two guest editors of this special issue: Susanna Donatelli and Jetty Kleijn. Moreover, I would like to thank all authors, reviewers, and the organizers of the Petri net conference satellite workshops, without whom this issue of ToPNoC would not have been possible.

November 2011

Kurt Jensen

Editor-in-Chief

LNCS Transactions on Petri Nets and Other Models of Concurrency (ToPNoC) 


\section{LNCS Transactions on Petri Nets and Other Models of Concurrency: Aims and Scope}

ToPNoC aims to publish papers from all areas of Petri nets and other models of concurrency ranging from theoretical work to tool support and industrial applications. The foundation of Petri nets was laid by the pioneering work of Carl Adam Petri and his colleagues in the early 1960s. Since then, an enormous amount of material has been developed and published in journals and books and presented at workshops and conferences.

The annual International Conference on Application and Theory of Petri Nets and Other Models of Concurrency started in 1980. The International Petri Net Bibliography maintained by the Petri Net Newsletter contains close to 10,000 different entries, and the International Petri Net Mailing List has 1,500 subscribers. For more information on the International Petri Net community, see: http://www.informatik.uni-hamburg.de/TGI/PetriNets/

All issues of ToPNoC are LNCS volumes. Hence they appear in all large libraries and are also accessible in LNCS Online (electronically). It is possible to subscribe to ToPNoC without subscribing to the rest of LNCS.

ToPNoC contains:

- revised versions of a selection of the best papers from workshops and tutorials concerned with Petri nets and concurrency;

- special issues related to particular subareas (similar to those published in the Advances in Petri Nets series);

- other papers invited for publication in ToPNoC; and

- papers submitted directly to ToPNoC by their authors.

Like all other journals, ToPNoC has an Editorial Board, which is responsible for the quality of the journal. The members of the board assist in the reviewing of papers submitted or invited for publication in ToPNoC. Moreover, they may make recommendations concerning collections of papers for special issues. The Editorial Board consists of prominent researchers within the Petri net community and in related fields.

\section{Topics}

System design and verification using nets; analysis and synthesis, structure and behavior of nets; relationships between net theory and other approaches; causality/partial order theory of concurrency; net-based semantical, logical and algebraic calculi; symbolic net representation (graphical or textual); computer tools for nets; experience with using nets, case studies; educational issues related to nets; higher level net models; timed and stochastic nets; and standardization of nets. 
Applications of nets to: biological systems, defence systems, e-commerce and trading, embedded systems, environmental systems, flexible manufacturing systems, hardware structures, health and medical systems, office automation, operations research, performance evaluation, programming languages, protocols and networks, railway networks, real-time systems, supervisory control, telecommunications, and workflow.

For more information about ToPNoC, please see: www.springer.com/lncs/ topnoc

\section{Submission of Manuscripts}

Manuscripts should follow LNCS formatting guidelines, and should be submitted as PDF or zipped PostScript files to ToPNoC@cs.au.dk. All queries should be addressed to the same e-mail address. 


\section{LNCS Transactions on Petri Nets and Other Models of Concurrency: Editorial Board}

\section{Editor-in-Chief}

Kurt Jensen, Denmark (http://person.au.dk/en/kjensen@cs.au.dk)

\section{Associate Editors}

Grzegorz Rozenberg, The Netherlands

Jonathan Billington, Australia

Susanna Donatelli, Italy

Wil van der Aalst, The Netherlands

\section{Editorial Board}

Didier Buchs, Switzerland

Gianfranco Ciardo, USA

José-Manuel Colom, Spain

Jörg Desel, Germany

Michel Diaz, France

Hartmut Ehrig, Germany

Jorge C.A. de Figueiredo, Brazil

Luis Gomes, Portugal

Roberto Gorrieri, Italy

Serge Haddad, France

Xudong He, USA

Kees van Hee, The Netherlands

Kunihiko Hiraishi, Japan

Gabriel Juhas, Slovak Republic

Jetty Kleijn, The Netherlands

Maciej Koutny, UK
Lars M. Kristensen, Norway

Charles Lakos, Australia

Johan Lilius, Finland

Chuang Lin, China

Satoru Miyano, Japan

Madhavan Mukund, India

Wojciech Penczek, Poland

Laure Petrucci, France

Lucia Pomello, Italy

Wolfgang Reisig, Germany

Manuel Silva, Spain

P.S. Thiagarajan, Singapore

Glynn Winskel, UK

Karsten Wolf, Germany

Alex Yakovlev, UK 


\section{Guest Editors' Preface}

This issue of ToPNoC contains revised versions of contributions selected from workshops and tutorial presentations held in conjuction with the 31st International Conference on Application and Theory of Petri Nets and Other Models of Concurrency, and the 10th International Conference on Application of Concurrency to System Design (ACSD 2010), co-located in Braga, Portugal, 21-25 June 2010; in addition it contains a contributed paper selected through the regular submission track of ToPNoC.

We are indebted to the Program Committees of the workshops and in particular their chairs. Without their enthusiastic work this volume would not have been possible. Many members of the Program Committees participated in reviewing the new versions of the papers selected for this issue. The strongest contributions were submitted from the following workshops:

- APNOC 2010: 2nd International Workshop on Abstractions for Petri Nets and Other Models of Concurrency

- ART 2010: Applications of Region Theory

- BioPPN 2010: International Workshop on Biological Processes and Petri Nets

- PNSE 2010: International Workshop on Petri Nets and Software Engineering

- SUMo 2010: Scalable and Usable Model Checking for Petri Nets and Other Models of Concurrency

The best papers of these workshops were selected in close cooperation with their chairs. The authors were invited to improve and extend their results where possible, based on the comments received before and during the workshop. The resulting revised submissions were reviewed by three to four referees. We followed the principle of also asking for fresh reviews of the revised papers, i.e., from referees who had not been involved initially in reviewing the original workshop contribution. All papers went through the standard two-stage journal reviewing process and eventually nine were accepted after rigorous reviewing and revising. This special issue is completed by two tutorial papers and by an article selected through the standard submission procedure of ToPNoC. Presented are a variety of high-quality contributions, ranging from model checking and system verification to synthesis, and from foundational work on specific net classes to innovative applications of Petri nets and other models of concurrency. 
Four papers of this issue are in the area of model-checking and state space exploration. The tutorial paper by Gianfranco Ciardo, Yang Zhao, and Xiaoqing Jin entitled Ten Years of Saturation: A Petri Net Perspective carefully reviews the use of decision diagrams to represent state spaces. The topic is tackled with particular attention to a technique for state space generation called saturation that was developed ten years ago in the context of Petri nets and that has gained wider applicability in the verification world. The result is an in-depth treatment that constitutes a not-to-be-missed bulk of knowledge on decision diagrams and Petri nets. The (regular) paper by Silien Hong, Fabrice Kordon, Emmanuel Paviot-Adet, and Sami Evangelista, Computing a Hierarchical Static Order for Decision Diagram-Based Representation from $P / T$ Net, addresses the well-known problem that the efficiency of decision diagrams may strongly depend on variable ordering. They show how the state space explosion problem can be dealt with for large Petri nets by mixing heuristics for variable ordering with a hierarchical form of decision diagrams. The work of Michal Knapik and Wojciech Penczek deals with Bounded Model Checking for Parametric Timed Automata. In parametric model checking, property verification relies on parameter synthesis. The paper shows how bounded model checking can be applied to parameter synthesis for parametric timed automata with continuous time leading to a theory useful for the parametric verification of a range of temporal formulae. Finally, the tutorial paper by Lars M. Kristensen and Michael Westergaard, $A$ Graphical Approach to Component-Based and Extensible Model Checking Platforms presents JoSEL, a graphical language for the specification of executable model checking jobs, which allows a user to work at different levels of abstraction when interacting with the underlying components of a model checking tool. The tutorial uses the model checking platform ASAP as a reference to illustrate the use of JoSEL with an existing model checking tool.

Another group of papers is concerned with synthesis, the construction of a system (e.g., a Petri net of a certain type) from a given behavioral specification. In Synthesis Problem for Petri Nets with Localities, Maciej Koutny and Marta Pietkiewicz-Koutny consider Petri nets with localities. In such nets the transitions are partitioned into disjoint groups within which execution is synchronous and maximally concurrent. This idea is generalized to overlapping causalities for which different semantical interpretations are given. It is shown how the resulting net classes, as instances of the general framework of $\tau$-nets, are synthesizable from behavioral specifications given in terms of finite step transition systems. Marc Solé and Josep Carmona, in their paper Incremental Process Discovery, are concerned with control-flow process discovery, meaning that given a representation of (part of) the behavior of a system in the form of a log (a set of observed sequences) or an automaton, an event-based formal model, here a Petri net, is generated as an underlying model for that behavior. Algorithms for this problem are often based on the theory of regions, but space requirements form an impediment for this approach. The paper discusses incremental derivation and exploration of a basis of regions, which makes it possible to split large inputs in fragments of tractable size. The resulting algorithm has been implemented in 
a tool that is experimentally tested and compared to other applications. As argued by Robin Bergenthum, Jörg Desel, Andreas Harrer, and Sebastian Mauser in Modeling and Mining of Learnflows, the area of workflow management has a lot in common with the field of learning and teaching processes (learnflows). Based on workflow modeling ideas, Petri net modeling languages for learnflows are introduced. Workflow mining methods are adapted to obtain a method for the synthesis of learnflow models from log files of learning systems.

Dorsaf Elhog-Benzina, Serge Haddad, and Rolf Hennicker discuss modal Petri nets (i.e., with distinguished may- and must-transitions) in Refinement and Asynchronous Composition of Modal Petri Nets. They provide procedures to decide whether such Petri nets are weakly deterministic and whether two modal language specifications generated by weakly deterministic modal Petri nets are related by the modal refinement relation. Asynchronously communicating modal I/O-Petri nets are studied as an application scenario.

Petri nets are gaining increasing attention as a formalism for the analysis of biological networks. The paper by Murad Banaji, Cycle Structure in SR and DSR Graphs: Implications for Multiple Equilibria and Stable Oscillation in Chemical Reaction Networks illustrates two graph-based formalisms, a labeled bipartite multigraph termed an SR graph, and its directed version, the DSR graph, for the analysis of oscillation behavior in chemical reactions. These objects are closely related to Petri nets, and the author discusses similarities and differences.

An innovative application of Petri nets to grid computing is the topic of the paper by Marco Mascheroni and Fabio Farina, Nets-Within-Nets Paradigm and Grid Computing. Nets-within-nets have been used to model the architecture of a Grid tool for High Energy Physics data analysis, and the paper provides evidence of how the modeling activity has led to the identification and solution of a number of defects in the tool implementation. Nets-within-nets can be found also in the paper Providing an Agent Flavored Integration for Workflow Management by Thomas Wagner, José Quenum, Daniel Moldt, and Christine Reese. It discusses the introduction of software agents, and their related concepts, into workflow management. Petri net based technologies are used to achieve a common basis for the integration and combination of workflow and agent technologies. The paper by Juan-Pablo López-Grao and José-Manuel Colom, A Petri Net Perspective on the Resource Allocation Problem in Software Engineering, starts from the observation that classical approaches to resource allocation analysis, as for flexible manufacturing systems, cannot naturally extend to software engineering, since the concept of resource is different (resource may be services that may be created or destroyed by the processes). The paper proposes therefore a generalization of the classical Resource Allocation Systems (RAS) classes, the usefulness of which is shown through the identification of a taxonomy of anomalies that can be found in the context of software systems. 
As guest editors, we would like to thank all authors and referees who have contributed to this issue: not only is the quality of this volume the result of the high scientific value of their work, but we would also like to acknowledge the excellent cooperation throughout the whole process that has made our work a pleasant task. Finally, we would like to pay special tribute to the work of Lars Madsen of Aarhus University who has provided technical support for the composition of this volume, including interactions with the authors. We are also grateful to the Springer/ToPNoC team for the final production of this issue.

November 2011

Susanna Donatelli Jetty Kleijn Guest Editors, Fifth Issue of ToPNoC 


\section{Organization}

\section{Guest Editors}

Susanna Donatelli, Italy

Jetty Kleijn, The Netherlands

\section{Co-chairs of the Workshops}

Natalia Sidorova (The Netherlands)

Alexander Serebrenik (The Netherlands)

Jörg Desel (Germany)

Alex Yakovlev (UK)

Claudine Chaouiya (Portugal)

Monika Heiner (Germany)

Michael Duvigneau (Germany)

Daniel Moldt (Germany)

Didier Buchs (Switzerland)

Fabrice Kordon (France)

Yann Thierry-Mieg (France)

Jeremy Sproston (Italy)

\section{Referees}

Wil van der Aalst

David Angeli

Eric Badouel

Marco Beccuti

Béatrice Bérard

Robin Bergenthum

Luca Bernardinello

Liu Bing

Josep Carmona

Ivana Cerna

Piotr Chrząstowski-Wachtel

Philippe Darondeau

Erik Flick

Giuliana Franceschinis

Serge Haddad

Xudong He

Kees van Hee
Vladimir Janousek

Peter Kemper

Victor Khomenko

Alex Kondratyev

Maciej Koutny

Lars Kristensen

Sebastian Mauser

Daniel Moldt

Marta Pietkiewicz-Koutny

Oana Prisecaru

Wolfgang Reisig

Charlotte Seidner

Natalia Sidorova

Jeremy Sproston

Enrico Vicario

Jan Martijn van der Werf

Mengchu Zhou 


\section{Table of Contents}

Cycle Structure in SR and DSR Graphs: Implications for Multiple

Equilibria and Stable Oscillation in Chemical Reaction Networks.......

Murad Banaji

Modeling and Mining of Learnflows $\ldots \ldots \ldots \ldots \ldots \ldots \ldots \ldots \ldots \ldots$

Robin Bergenthum, Jörg Desel, Andreas Harrer, and

Sebastian Mauser

Ten Years of Saturation: A Petri Net Perspective

Gianfranco Ciardo, Yang Zhao, and Xiaoqing Jin

Refinement and Asynchronous Composition of Modal Petri Nets . . . . . .

Dorsaf Elhog-Benzina, Serge Haddad, and Rolf Hennicker

Computing a Hierarchical Static Order for Decision Diagram-Based

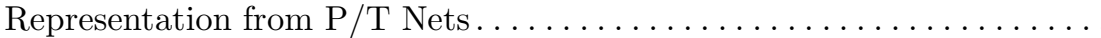

Silien Hong, Fabrice Kordon, Emmanuel Paviot-Adet, and

Sami Evangelista

Bounded Model Checking for Parametric Timed Automata . . . . . . . . . 141

Michat Knapik and Wojciech Penczek

Synthesis Problem for Petri Nets with Localities

Maciej Koutny and Marta Pietkiewicz-Koutny

A Petri Net Perspective on the Resource Allocation Problem in

Software Engineering . . . . . . . . . . . . . . . . . . .

Juan-Pablo López-Grao and José-Manuel Colom

Nets-Within-Nets Paradigm and Grid Computing ..............

Marco Mascheroni and Fabio Farina

Incremental Process Discovery . . . . . . . . . . . . . . . .

Marc Solé and Josep Carmona

Providing an Agent Flavored Integration for Workflow Management ....

Thomas Wagner, José Quenum, Daniel Moldt, and Christine Reese

A Graphical Approach to Component-Based and Extensible Model

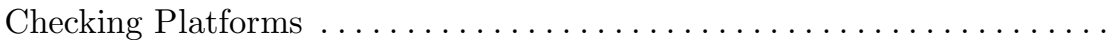

Michael Westergaard and Lars Michael Kristensen

Author Index 